\title{
Evidence-based perioperative risk reduction
}

\author{
W. Scott Beattie MD PhD FRCPC
}

$\mathrm{T}$

HE purpose of this article is to examine the perioperative cardiac risk evidence and comment on the recommendations stemming from the evidence. We will assess the effect the evidence and the recommendations have had on practice.

\section{The dilema}

Have the changes made to perioperative practice modified outcomes?

Death is four times more common on the first day after surgery than the death rate in an age and risk adjusted population not having surgery. ${ }^{1}$ More than $50 \%$ of these deaths are a direct result of cardiovascular insult. The hospital costs for a postoperative cardiac related death double. ${ }^{2}$ The costs of a postoperative myocardial infarction (MI) are 50\% higher than uncomplicated surgery. Furthermore, patients who survive a postoperative MI, are twice as likely to die in the next two years as patients having uncomplicated surgery. ${ }^{3}$ The last decade has seen the introduction of preoperative assessment strategies, the America College of Cardiology and the American Heart Association (ACC/AHA) guidelines, new drugs, and minimally invasive surgical techniques. With these "improvements" there is no evidence that we have been able to alter postoperative mortality rates.

Are we doing something wrong?

\section{The perioperative assessment}

We undertake risk stratification in the hope that by doing so we can institute therapy that will favourably alter outcome. This implies that the strategies are accurate, and once identifying a high-risk patient, that there are effective modes to reduce risk.

\section{Is the preoperative assessment accurate?}

The delineation of risk begins with a good history and physical examination. There are several preoperative assessment strategies published. These strategies are not widely employed in clinical practice, most being too cumbersome or hard to remember. Table I com- pares three of the available risk indices. The revised cardiac risk index (RCRI) ${ }^{4}$ is the most accurate and the easiest to remember. The RCRI has now been employed in several clinical trails to stratify for risk. We need to adopt the RCRI more widely.

The risk indices perform best when coupled with the results of preoperative cardiac testing. The easiest test is a routine electrocardiogram (EKG). The finding of any abnormality in high-risk patients triples the perioperative risk. A normal EKG in a low risk patient has little discrimination. A 2D echocardiograph can be used in many ways to discriminate risk. The full potential of resting 2D echocardiogram has not been exploited at present. There is good evidence that estimation of ventricular function delineates risk. ${ }^{5-7}$ The finding of mitral regurgitation has also been linked to poor cardiac outcomes. ${ }^{6}$ Finally, the demonstration of aortic stenosis has been shown to increase perioperative cardiac risk. ${ }^{8}$

The ACC/AHA guidelines suggest that the best test, for preoperative cardiac risk stratification, is an exercise EKG. However, the demonstration of ST depression in women is not as sensitive or specific as it is in men, $30 \%$ of patients have resting abnormalities which render the test non-specific, and many cannot exercise due to orthopedic and/or peripheral vascular limitations. The ACC/AHA guidelines suggest the use of persantine-thallium/sestamibi (DTI ) in these situations. The accuracy of DTI is summarized in five previous meta-analyses. ${ }^{9-13}$ Etchells et al. ${ }^{10}$ in a most eloquent analysis, show that a negative test DID NOT reduce the likelihood ratio of an event (that is a high proportion of test were false negative tests). The perfusion defect had to measure greater than $30 \%$ of left ventricular (LV) mass before the positive test significantly changed the likelihood ratio of a postoperative cardiac event. A recently completed meta-analytic comparison of DTI and dobutamine stress echocardiography (DSE) found that false negative test occurs more than twice as often with DTI than with DSE. The receiver operating curves confirms that DSE is more accurate (Figure 1). We have concluded that the

From the Department of Anesthesia, University Health Network, University of Toronto, Toronto, Ontario, Canada.

Address correspondence to: Dr. W. Scott Beattie, R. Fraser Elliott Chair in Cardiac Anesthesia, Department of Anesthesia, University Health Network, University of Toronto, 200 Elizabeth Street, Toronto, Ontario M5G 2C4, Canada. Phone: 416-340-4800;

Fax: 416-340-3698; E-mail: scott.beattie@uhn.on.ca 
TABLE I Comparison of three risk indices

\begin{tabular}{lllll}
\hline & $n$ & $\begin{array}{l}\text { Goldman rish index } \\
\text { ROC }(95 \% \text { CI })\end{array}$ & $\begin{array}{l}\text { Detsky modified } \\
\text { ROC (95\% CI) }\end{array}$ & Revised cardiac risk index \\
\hline Goldman & 1001 & $0.810(0.02)$ & & \\
Detsky & 455 & $0.800(0.08)$ & & \\
Zeldin & 1140 & $0.606(0.03)$ & $0.545(0.02)$ & $0.759(0.03)$ \\
Lee (derivation set) & 2893 & $0.701(0.04)$ & $0.582(0.03)$ & $0.806(0.03)$ \\
Lee (validation set) & 1422 & $0.620(0.03)$ & $0.630(0.03)$ & $0.730(0.03)$ \\
Kumar & 1000 & $0.643(0.05)$ & $0.601(0.06)$ & $0.793(0.04)$ \\
Gilbert & 2035 & & $0.814(0.06)$ & \\
Beattie & 1000 & & & \\
\hline
\end{tabular}

ROC $=$ receiver operative characteristic.

recommended screening tool is not the most accurate. Stress echo is not readily available in most hospitals even though stress echo costs less, requires less infrastructure, and it is more convenient for patients.

Does using a less accurate screening tool affect outcomes? Presently, there is no way of knowing what the effect of a false negative test is; we speculate that a negative test will give a sense of security, and in turn decrease the employment of useful risk reduction strategies. The increased false positive rate, seen with DTI, has lead to significantly more coronary angiography without a concomitant increase in revascularization. ${ }^{14}$

The evidence supports the increased utilization of stress echo for preoperative screening. In addition this test will evaluate resting ventricular function, wall motion abnormalities, and valve function.

Having identified a high-risk patient, can we reduce perioperative cardiac events?

\section{Perioperative risk reduction}

\section{Prophylactic coronary revascularization}

Coronary artery surgery decreases mortality, in patients with left main coronary disease, and in patients with three-vessel disease and poor ventricular function. It stands to reason that this benefit will also be seen in patients coming for non-cardiac surgery. Conceptually, the prophylactic use of revascularization subjects a patient to increased surgical risk on two separate occasions. Since we lack clear direction, two separate decision analyses have been conducted. ${ }^{15,16}$ Both analyses show that, the benefit of prophylactic revascularization only applies benefit when the risk of [coronary] angiography and subsequent coronary revascularization is $2 \%$ less than the risk of a cardiac event occurring during a non-cardiac surgical procedure under medical therapy alone. One of the studies suggests that the greatest benefit accrues when angiography reveals a risk is so high that the non-cardiac surgery is cancelled. ${ }^{16}$

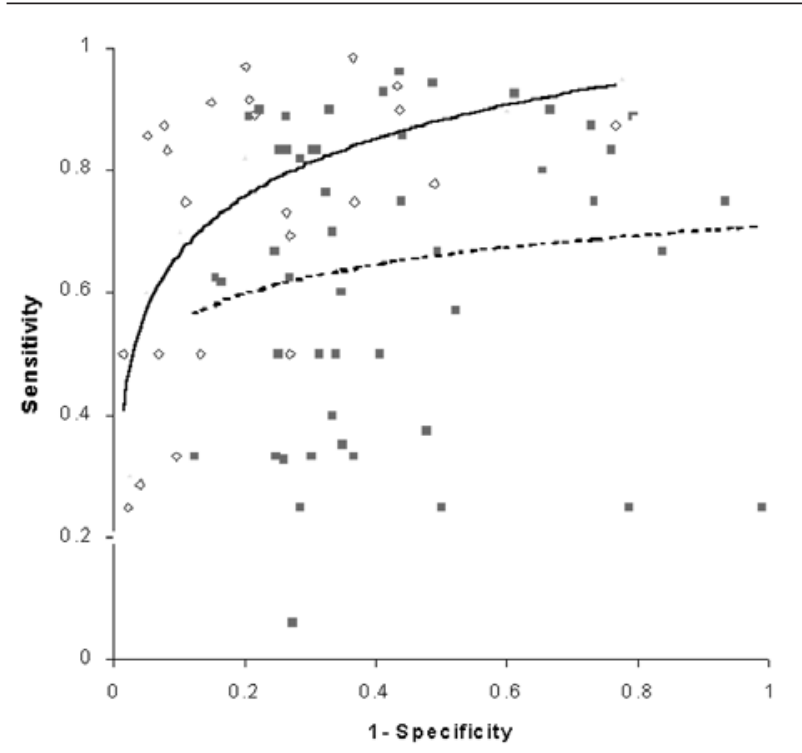

FIGURE 1 Graph showing the summary receiver operative characteristic (SROC) for the 25 stress echocardiography studies or the 47 stress nuclear scintigraphy studies. The solid line represents the SROC for echocardiography while the dotted line represents the stress scintigraphy SROC. The stress echocardiography line is shifted higher and to the left showing superior delineation.

Which is the best method of revascularization?

There has never been an adequate discussion of whether the type of revascularization coronary artery bypass grafting (CABG) ps percutaneous coronary intervention (PCI) is an important factor. The benefit of PCI, in the short term, is that the risk of stroke is decreased sixfold in PCI patients compared to CABG. ${ }^{17}$ Landesberg et al., in an observational propensity matched study, ${ }^{\mathrm{A}}$ have shown that $\mathrm{CABG}$ is superior to PCI for long-term outcomes. ${ }^{18}$ The benefit was not apparent until three to four years after surgery. In this study, revascularization procedures 
TABLE II In hospital cardiac morbidity comparing prophylactic revascularization to medical therapy

\begin{tabular}{|c|c|c|c|c|c|c|}
\hline \multirow[t]{2}{*}{ Study } & \multirow[b]{2}{*}{ Revascularization } & \multicolumn{2}{|l|}{ Myocardial infarction } & \multirow[b]{2}{*}{ Revascularization } & \multicolumn{2}{|l|}{ Death } \\
\hline & & No revascularization & $\begin{array}{l}\text { Effect ( } 95 \% \\
\text { confidence interval) * }\end{array}$ & & No revascularization & Effect (95\%) \\
\hline Eagle et al. & $8 / 964$ & $16 / 582$ & $0.30(0.13,0.70)$ & $17 / 964$ & $19 / 582$ & $0.53(0.27,1.03)$ \\
\hline Posner et al. & $15 / 686$ & $19 / 686$ & $0.79(0.38,1.63)$ & $18 / 686$ & $20 / 686$ & $0.90(0.46,1.77)$ \\
\hline PTCA (< 90 days & $10 / 142$ & $5 / 142$ & $2.0(1.5,2.5)$ & $3 / 142$ & $4 / 142$ & $.75(0.5,2.0)$ \\
\hline $\begin{array}{l}\text { PTCA (> } 90 \text { days) } \\
\text { Hassan } \text { et al. (BARI) }\end{array}$ & $5 / 544$ & $14 / 544$ & $0.33(0.22,0.67)$ & $15 / 544$ & $16 / 544$ & 1 \\
\hline CABG & $2 / 250$ & & NA & $2 / 250$ & & NA \\
\hline Angioplasty & $2 / 251$ & & NA & $2 / 251$ & & NA \\
\hline Kaluza et al. & & & & & & \\
\hline $\begin{array}{l}\text { Stent }<40 \text { days } \\
\text { Abdelnaem } e t a l .\end{array}$ & $7 / 40$ & NA & $9 / 40$ & & & NA \\
\hline $\begin{array}{l}24 \text { studies } \\
\text { (1,097 patients) }\end{array}$ & $7 / 165 /$ & $178 / 932$ & $0.43(.025,76)$ & & & NA \\
\hline $\begin{array}{l}\text { McFalls et al. } \\
\text { CARP trial } \\
\text { (mixed PCI and CABG) }\end{array}$ & $30 / 258$ & $36 / 252$ & $0.79(0.47-1.33)$ & $8 / 258$ & $9 / 252$ & $.86(33,2.28)$ \\
\hline
\end{tabular}

PTCA = percutaneous transluminal coronary angioplasty; $\mathrm{CABG}=$ coronary artery bypass graft; CARP $=$ coronary artery revascularization prophylaxis; $\mathrm{PCI}=$ percutaneous coronary intervention.

were superior to medical therapy. Table II presents the in-hospital cardiac events in the publications reviewing prophylactic revascularization preceding non-cardiac surgery. The invasive treatment of coronary disease continues to evolve. The data on elective PCI preceding elective non-cardiac surgery suggest that if the interval between angioplasty and non-cardiac surgery is shorter than six weeks, PCI will increase the perioperative cardiac event rate (Table II). The data from the Kaluza $^{19}$ and Posner ${ }^{20}$ trials are particularly interesting in this aspect of perioperative care. The early results from BARI show diabetics have a much higher mortality after PCI. ${ }^{17}$ The newer PCI and stent technology requires prolonged use of anticoagulant and antiplatelet therapy, which will increase the interval between diagnosis and surgery. Coronary artery surgery also continues to improve. There is now evidence that off-pump coronary artery surgery $(\mathrm{OPCAB})$, in high-risk patients, reduces perioperative stroke, MI, and atrial fibrillation. OPCAB surgery shows no difference in death rate at one year but there is almost a twofold increase in the need for further revascularization. ${ }^{21}$ In short, we don't know which is the best method of revascularization.

A A propensity analysis is a statistical tool that calculates the probability of an event (the chance of being on a betablocker) then matching patients in the data base who have the same probability of the event, but in whom the event did not take place (not being prescribed a beta-blocker). This matching technique sets up a situation which is similar to a randomization.
Does prophylactic revascularization reduce non-cardiac surgical risk?

The results of the coronary artery revascularization prophylaxis (CARP) trial were recently reported (as an abstract). The trial is not widely applicable to our practice. This trial was conducted in the Veteran's Administration (VA) system, therefore it is mostly a male population. All patients were having elective vascular surgery. All patients were randomized after coronary angiography and all patients with main left disease and poor LV function were excluded. The medical arm of the trial was not defined. Twenty-five percent of the randomized patients were not high-risk and therefore were not the type of patients we would consider for angiography. More patients had PCI (60 vs 40) than CABG. The incidence of morbid cardiac events, during the revascularization phase of the trial, was $1.7 \%$. There was no difference in death rate after two years. There was a $25 \%$ reduction in the in-hospital MI rate, which did not achieve significance, but the trial was underpowered to detect such a difference. There was no difference in the long-term results; events in $23 \%$ of the medical therapy group and $22 \%$ of the revascularized group. These results are similar to the mortality rates reported by Landesberg. ${ }^{18}$

A meta-analysis of studies assessing preoperative stress testing ${ }^{14}$ shows that when the test is positive, and in turn leads to a coronary angiogram, there is a significant reduction in the in-hospital cardiac events. This meta-analysis could not separate the patients who had left main disease or poor ventricular function 
however; the magnitude of reduction in the in-hospital infarction rate demonstrated in the CARP trial is similar to that found in our meta-analysis.

By convention, we continue to offer prophylactic coronary revascularization to patients with left main coronary disease, and three-vessel disease with poor LV function. The CARP trial does not offer any insight on how to screen high-risk patients for left main disease. In moderate to high-risk patients with less severe coronary disease, prophylactic revascularization may offer up to a $25 \%$ reduction in in-hospital MI, but little in the way of long-term benefit.

"What is important about these findings are the benefits of optimal medical therapy. "I'd like to underscore the importance of medical therapy, such as beta blockers at the time of vascular surgery."

Dr. Don Poldermans commenting on the CARP results at the AHA meeting 2004.

\section{Medical therapy}

Perioperative beta blockade

"When possible, beta blockers should be started days or weeks before elective surgery, with the dose titrated to achieve a resting heart rate between 50 and 60 beats. $\mathrm{min}^{-1}$."

ACC/AHA practice guidelines - 2002.

"A large percentage of postoperative MI may be preventable through improved use of $\beta$-blockers perioperatively. Greater efforts should be made to encourage $\beta$-blocker use among high-risk patients undergoing major non-cardiac surgery."

Arch Intern Med 2004; 164: 762-6.

These quotes represent the conventional wisdom and form the basis of medical perioperative risk reduction. Everyone has heard, about the evidence from Mangano $^{22}$ then Poldermans ${ }^{23}$ studies. Both [of these] studies have design flaws. The Mangano study did not show any beta-blocker effect on the in-hospital cardiac event rate. Patients having in-hospital cardiac events were excluded from the long-term results. The Poldermans study was unblinded, and in very high-risk vascular patients. Entry criteria were for patients having a RCRI score of greater than 2 and who demonstrate new wall motion abnormalities on preoperative stress echocardiography. The Safety Committee stopped the trial early. Two meta-analy$\operatorname{ses}^{24,25}$ have shown the cumulative effect, in just over 1,000 patients, was a relative risk reduction of $40 \%$. In

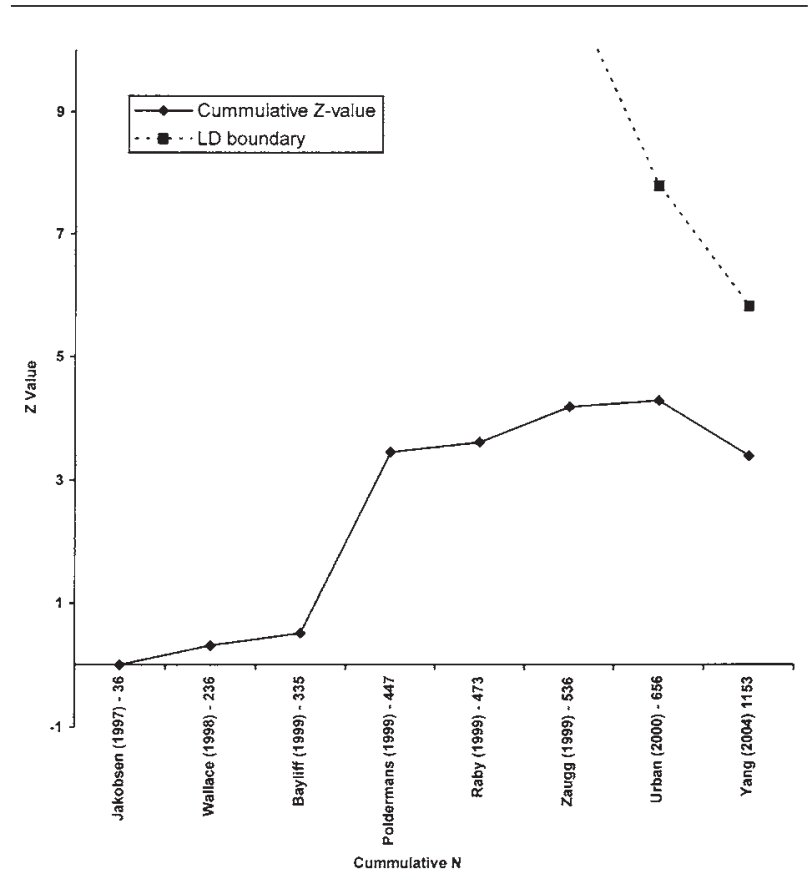

FIGURE 2 Cumulative meta-analysis assessing the effect of perioperative beta-blockers on the 30-day risk of major perioperative cardiovascular events (i.e., cardiovascular death, nonfatal myocardial infarction, or nonfatal cardiac arrest) in patients undergoing non-cardiac surgery. The Lan-DeMets (LD) sequential monitoring boundary, that assumes $10 \%$ control event rate and a $25 \%$ relative risk reduction with $80 \%$ power and a two-sided alpha $=0.01$, has not been crossed indicating that the cumulative evidence is inconclusive. The points along the $\mathrm{x}$ axis denote each separate study, the year it was carried out, and the cumulative number of patients included in the analysis.

the last year, two, well-designed, larger trials, in at risk patients, $\left[(\mathrm{MaVS})^{26}\right.$ and DIPOM] did not show any benefit from perioperative beta-blockers

In a recently completed meta-analysis, (which includes the data from MaVS and DIPOM) Devereaux et al., indicates that the relative risk of a perioperative cardiac event after beta-blocker therapy is $0.83[95 \%$ confidence interval (CI) $0.44-1.58 P=.52$ ]; (Figure 2 ). If the bisoprolol study is removed there is no effect of perioperative beta-blockers in the short term. Interestingly, the risk reduction found in this metaanalysis is the same rate that Lindenauer $e t$ al. report in a risk adjusted database analysis of over 750,000 patients (currently in press). In that analysis, based on matching by propensity score, perioperative use of beta blockers was associated with reduced in-hospital mortality $[2.78 \%$ vs $3.38 \% P<0.001$, odds ratio (OD) 0.82 $(95 \%$ CI $0.77,0.86)]$. This benefit varied directly with 
cardiac risk; for patients with RCRI $1-4$ or higher the adjusted OD and 95\% CI for in-hospital mortality were $0.94(0.88,1.01), 0.82(0.77,0.88), 0.72(0.64,0.81)$, $0.63(0.62,0.76)$. Note that the highest risk group approaches the results of the Polderman trial.

In a fourth meta-analysis, Giles et al. ${ }^{27}$ could not demonstrate a positive effect in patients coming to surgery taking chronic beta-blockers. Indeed, it is known that patients chronically on a beta-blocker probably go through a withdrawal phenomenon since postoperative patients cannot take oral medications. Beta-blocker withdrawal can induce ischemia and/or arrhythmias, leading to a prolonged length of stay. This is particularly evident after cardiac surgery. The highest incidence of atrial fibrillation, documented in a large clinical trial, after cardiac surgery was found in patients on chronic beta-blocker therapy who were randomized to receive placebo. ${ }^{28}$ There are no data to quantify how often beta-blocker withdrawal occurs or the magnitude of the effect. During the startup of the POISE $^{B}$ trial it was found that few hospitals had a postoperative protocol for administering iv betablocker. We need to make every attempt to continue beta-blockers throughout the perioperative period.

The evidence to date is consistent and suggests that beta-blockers reduce perioperative cardiac events in high-risk patients. There is no evidence to suggest that beta-blockers are helpful in low to moderate risk patients. As clinicians we need to be mindful of betablocker withdrawal, and make every effort to prevent it.

"An ongoing large randomized trial involving 10,000 patients will test whether beta-blockers are useful in lower risk patients."

Dr. Lee Goldman evidence based perioperative risk reduction Am J Med 2003.

\section{Alpha ${ }_{2}$ adrenergic agonists}

This class of drugs received a llb recommendation in the 2002 ACC/AHA guidelines (usefulness is less well established by evidence/opinion).

In a subsequent meta-analysis ${ }^{29}$ alpha $_{2}$ agonists were found to reduce the incidence of postoperative ischemia and MI. Since the publication of the metaanalysis a randomized trial using transdermal clonidine has been published documenting a reduction in postoperative MI and death. ${ }^{30}$ There are now over 2,000 patients who have received alpha ${ }_{2}$ agonists in trials and

B POISE is the CIHR funded investigation of perioperative metoprolol in moderate to high-risk non-cardiac surgical patients. have documented 83 perioperative cardiac events. The relative risk for all alpha ${ }_{2}$ agents is 0.59 (95\% CI 0.34-0.93). Clonidine, the oldest and cheapest of these drugs, also appears to have the best results.

Clonidine has sedative properties, and analgesic properties. It reduces morphine requirements ${ }^{31}$ when administered intravenously and centrally. There is also a reduced incidence and severity of postoperative nausea and vomiting. There are relatively few side effects and virtually no new instances of congestive heart failure. The side effects include hypotension and bradycardia, these effects occur but in the same proportion as with beta-blockade.

The evidence in support of clonidine for perioperative risk reduction is as strong as that for beta-blockers. The major problem for clinicians in Canada is there are few formulations and little if any use of these agents at the present time.

\section{Calcium channel blockers}

"There bave only been two studies examining the role of calcium channel blockers in this situation. These studies are too small to allow definitive conclusions."

\section{ACC/AHA Guidelines 2002.}

"To those who favor CCB's rather than betaadrenergic receptor blockers, we say: Provide the scientific evidence."

Butterworth and Furberg "Improving outcomes after non-cardiac surgery". Anesth Analg 2003.

There is some hesitation to use calcium channel blockers, stemming from the initial problems with the dihydropyridines. Drugs like nifedipine were shown to increase the MI rates in stable angina patients. Dihydropyridines are primarily vasodilators and have no negative chronotropic effect. A recent meta-analysis assessing the effect of calcium channel blockers ${ }^{32}$ identified 11 trials including 1,007 patients. All studies were published prior to 2002. The analysis found that calcium channel blockers reduced the incidence of ischemia; postoperative supraventricular tachycardia (SVT); (OR 0.52 95\% CI 0.3-.8), MI and major morbid events (0.39 95\% CI 0.17-0.89). The effects were almost entirely due to the experience with diltiazem. The dihydropyridine class of calcium channel blockers was found to increase ischemia, and the incidence of postoperative SVT.

All trials in this meta-analysis were testing the calcium channel blockers as single agents. We know little of the combination of calcium channel with other drugs. 


\section{Nitrates}

Nitroglycerine, the oldest of the therapies, has relatively few trials to compare. A recently completed meta-analysis, published in the Cochrane database, documents the experience of perioperative nitroglycerine in 11 trials (seven in cardiac surgery ) and 1,001 patients. There are too few studies in non-cardiac surgery to comment on MI or death. Nitrates have shown a clear improvement in perioperative ischemia over placebo. (OR 0.52 (95\%CI $0.31-0.88)$ ). In this analysis nicorandil seems to have superior properties to the other nitrates. The effects of nitrates have also been compared to calcium channel blockers in six cardiac surgery trials. The relative risk of ischemia was 0.51 (95\% CI 0.33-0.77) favouring the calcium channel blockers. The effect on MI was similar but underpowered to show a significant effect [OR $0.62(95 \%$ CI 0.24-1.64)].

\section{Anti-platelet agents}

This class of agents is an important aspect of the treatment of coronary artery disease. However, the ACC/AHA guidelines did not comment on the two largest perioperative studies pertaining to the protective effects of aspirin.

The $\mathrm{ACE}^{33}$ trial was a randomized controlled trial of varied doses of ASA given preoperatively to 2,849 patients having elective carotid endarterectomy. This trail found that low-dose ASA (less than $325 \mathrm{mg}$ per day) was associated with a $22 \%$ reduction in MI stroke or death. The trial was complicated by the fact that many patients were already on a dose of ASA and there was no time to have them withdraw from their current therapy. In an efficacy analysis of the trial, 1,700 of the randomized patients, who were on a prior dose of ASA, were excluded from the analysis. The effect of low dose aspirin, in the efficacy analysis is a $54 \%$ reduction in the incidence of stroke MI or death. The clear suggestion is that ASA in doses above $325 \mathrm{mg}$ are harmful.

The other major trial that was not referenced in the AHA guidelines was the pulmonary embolism prevention (PEP) trial. ${ }^{34}$ This was a trial in emergency or elective hip and knee surgery comparing $162 \mathrm{mg}$ ASA perioperatively to placebo. This trial found that there was a $36 \%$ reduction in thromboembolic events with this low dose of aspirin. Again, (in this trial) there was a suggestion that the antiplatelet effect was diminished if this dose was combined with preoperative nonsteroidal anti-inflammatory drugs (NSAIDs). Neither trial shows increased bleeding, nor was there any increased need for blood transfusion, when these low doses of aspirin were administered perioperatively.
A previously published meta-analysis of perioperative ASA did not account for the difference in doses. ${ }^{35}$ We have reanalyzed the data from this meta-analysis, applied dosage criteria, and excluded trials where we could not account for the concomitant use of other NSAIDS. We found that at a dose greater then 650 mg. day ${ }^{-1}$, ASA is associated with a threefold increase in MI. Alternatively, if the dose of aspirin was below 325 $\mathrm{mg} \cdot \mathrm{day}^{-1}$ there is a $50 \%$ reduction in perioperative MI.

This interpretation of this data is new; it has not been subject to peer review and should be treated as such. Aspirin in low doses appears safe, and does not increase blood transfusions. Perioperative low-dose aspirin appears to be cardioprotective. The use of NSAIDs, as adjuncts to analgesia, in patients at risk for cardiac events is not prudent until there is more evidence.

\section{Statins}

The first study to suggest a beneficial effect of lipid lowering therapy did not appear until 2003. Since that time, two observational studies ${ }^{36,37}$ with appropriate statistical risk stratification have shown a $30 \%$ reduction on postoperative cardiac events. One randomized controlled trial, in 100 vascular patients, assessing the effect of $20 \mathrm{mg}$ atrovastatin, ${ }^{38}$ started seven days preoperatively was associated with a $50 \%$ reduction in inhospital mortality. The long-term (six months) beneficial effects of therapy were also found.

The study by Lindenauer et al. ${ }^{36}$ deserves special attention. This is an observational database; a propensity score was developed to determine the likelihood of using a statin in the first two days after surgery. The database was then queried to match patients with the same likelihood of receiving a statin, but who did not. This then achieved a quasi-randomized trial in over 140,000 patients. The risk of perioperative death was reduced by $30 \%$ in patients who had a statin started within two days of surgery. The RCRI was used to indicate perioperative risk and the protective effect of statins was more pronounced in higher risk patients.

\section{Regional anesthesia}

"Most important, an effective analgesic (i.e. one that blunts the stress response) regimen must be included in the perioperative plan."

ACC/AHA Guidelines 2002.

"We were unable to find any significant improvement in major morbidity or mortality after major abdominal surgery from perioperative epidural analgesia."

The MASTER Trial Investigators 2003. 
In the only double-blind randomized trial conducted in perioperative epidural anesthesia Breslow et al. ${ }^{39}$ show a reduction in the stress response for $24 \mathrm{hr}$ with a single dose of epidural morphine. No fewer than four separate meta-analyses, ${ }^{38,40-42}$ outlining the effects of perioperative regional anesthesia have been published in the last decade. These studies have demonstrated superiority in the analgesia, respiratory complications and cardiac complications. Since the publication of the last of these meta-analyses four apparently negative trials have been published. The MASTER trial and the VA cooperative trial have suggested that there was very little cardiac benefit. Even though these are the largest trials to date, each enrolling almost 1,000 patients, they were underpowered to show a cardiac or death benefit. When these trials were used to update the cardiac meta-analysis, thoracic epidural continues to show a statistical significant reduction in MI relative risk (RR) 0.57 (95\% CI $0.37-0.89 P>0.01)$. Rodgers has used these newer trials to update their meta-analysis, in over 11,000 patients. The benefit is carried through to reduce mortality OR $0.78(0.63-0.97)$ reduce the incidence of pneumonia, OR $0.61(0.53-0.73)$ and $\mathrm{MI}$, OR $0.71(0.51-0.98)$.

The balance of evidence continues to support wider use of regional anesthesia.

\section{Comparison of prophylactic agents}

There are no trials, which compare the efficacy of any of these agents. Tables III and IV compare results obtained from the series of meta-analyses. The efficacy of the sympatholytic agents (beta-blockers, alpha $_{2}$ agonists and thoracic epidural) in preventing MI and death are remarkably similar. The side effect profiles are seen in Table IV. Bradycardia, requiring treatment, is seen with beta-blockers. Hypotension requiring treatment is seen with epidural use. The significance of either of these events is unknown given that MI and ischemia are improved. Low-dose aspirin did not cause a significant increase in the need for transfusion. Epidural analgesia is associated with lower transfusion requirements. There is a suggestion from this data that congestive heart failure is most frequent with beta-blockers.

At the present time, the balance of evidence supports the increased use of alpha ${ }_{2}$ agonists.

\section{Combination therapy}

The treatment of coronary disease and angina relies on multimodal therapy. There are no prospective trials documenting multiple therapies in non-cardiac surgery. Two publications of data from two prospec- tive databases have compared the additive effects of beta-blockers and lipid lowering agents. Both publications show that the effect of single therapy is dependant on the preoperative risk. That is, there is little or no benefit in patients with a preoperative RCRI score of 1 whereas in patients with a RCRI of 3 there is approximately a $50 \%$ risk reduction. The addition of a second agent further reduces risk another $50 \%$ in the high-risk patients. Neither study found an appreciable effect in the low-risk population, in fact there is a suggestion of increased side effects in the low-risk population. The concept of multiple drug therapy (utilizing separate classes of drugs) in high-risk populations requires more investigation.

\section{Conduct of the anesthetic}

Does the type of anesthetic influence the cardiac outcome?

For many years anesthesiologists have argued whether the type of anesthetic influenced outcome. Years ago we assumed the discussion was closed. However, with the emergence of the concept of ischemic preconditioning the issue may be re-emerging and important to our discussion. The inhalational anesthetics are thought to possess ischemic preconditioning properties. ${ }^{41}$ Most investigations of anesthetic ischemic preconditioning have been done in cardiac anesthesia, but the potential effects in the high-risk cardiac patients having non-cardiac surgery deserves consideration. In a meta-analysis of 19 studies including 20,000 patients there was no net reduction in the inhospital MI rate. However, there was significant heterogeneity in the analysis. Two agents, enflurane and halothane, were associated with increased MIs. When enflurane and halothane are excluded from the metaanalysis, isoflurane, sevoflurane, or desflurane, collectively show a $38 \%$ reduction in MI. This preliminary work requires more investigation.

Two independently conducted trials ${ }^{43,44}$ have shown nitrous oxide to increase the incidence of perioperative and postoperative myocardial ischemia. Furthermore, nitrous oxide increases homocysteine levels for over $72 \mathrm{hr}$ postoperatively. This may influence both platelet reactivity and endothelial function. Nitrous oxide has the added distinction of doubling the incidence of postoperative nausea and vomiting. Moreover, nitrous oxide is a poor anesthetic agent.

There is no reason to use nitrous oxide in patients at high risk for postoperative cardiac complications. 
TABLE III Comparison of the efficacy of single agent medical therapies

\begin{tabular}{|c|c|c|c|c|c|c|c|}
\hline \multirow[t]{2}{*}{ Study } & \multicolumn{4}{|c|}{ Death (incidence) } & \multicolumn{3}{|c|}{ Myocardial infarction (incidence) } \\
\hline & Trials & Treat & Cont & Effect $(95 \% C I)^{*}$ & Treat & Cont & Effect $(95 \% C I)^{*}$ \\
\hline \multicolumn{8}{|l|}{ Beta-blockers } \\
\hline $\begin{array}{l}\text { Devereaux et al. } \\
\text { Calcium channel blockers }\end{array}$ & 10 & $9 / 475$ & $19 / 475$ & OR $0.63(0.25-1.54)$ & $58 / 1052$ & $63 / 1021$ & RR $0.83(0.44-1.58)$ \\
\hline $\begin{array}{l}\text { Wijeysundera } e t a l . \\
\text { Alpha }_{2} \text { agonists }\end{array}$ & 7 & $5 / 358$ & $12 / 334$ & RR $0.40(0.14-1.16)$ & $0 / 252$ & $5 / 234$ & RR $0.25(0.05-1.12)$ \\
\hline $\begin{array}{l}\text { Wijeysundera } \\
\text { (updated Wallace et al.) } \\
\text { Nitrates }\end{array}$ & 9 & $30 / 1514$ & $45 / 1337$ & RR $0.61(0.39-0.96)$ & $83 / 1486$ & $93 / 1313$ & RR $0.84(0.64-1.12)$ \\
\hline Bender $e t a l . \dagger$ & 2 & $0 / 31$ & $0 / 29$ & NA & $0 / 53$ & $1 / 52$ & OR $0.33(0.01-8.63)$ \\
\hline $\begin{array}{l}\text { Thoracic epidural } \\
\text { Beattie et al. }\end{array}$ & 9 & $29 / 937$ & $41 / 933$ & RR $0.69(0.42-1.12)$ & $29 / 815$ & $52 / 820$ & RR $0.53(0.35-0.88)$ \\
\hline $\begin{array}{l}\text { Low dose aspirin } \\
\text { Unpublished }\end{array}$ & 4 & $547 / 12575$ & $577 / 12552$ & RR $0.94(0.84-1.05)$ & $93 / 10529$ & $114 / 10511$ & RR $0.79(0.60-1.05)$ \\
\hline
\end{tabular}

$\mathrm{CI}=$ confidence interval $\mathrm{RR}=$ relative risk $\mathrm{NA}=$ data not available.

TABLE IV Side effect profiles of the proposed perioperative risk reduction therapies

\begin{tabular}{llllll}
\hline & $\begin{array}{l}\text { Hypotension OR } \\
(95 \% \text { CI })\end{array}$ & $\begin{array}{l}\text { Bradycardia OR } \\
(95 \% \text { CI })\end{array}$ & $\begin{array}{l}\text { Congestive heart } \\
\text { failure OR (95\% CI) }\end{array}$ & $\begin{array}{l}\text { Bronchospasm } \\
\text { OR (95\% CI) }\end{array}$ & $\begin{array}{l}\text { Bleeding complications } \\
\text { requiring transfusion } \\
\text { OR (95\% CI) }\end{array}$ \\
\hline Beta blocker & $1.41(0.87-2.29)$ & $2.27(1.5-3.3)$ & $1.54(0.8-2.6)$ & $0.84(0.5-1.4)$ & NA \\
Alpha $_{2}$ agonist & $1.04(0.9-1.21)$ & $1.05(0.82-1.36)$ & $0.82(0.5-1.4)$ & NA & NA \\
Calcium channel blocker & $3.32(0.7-15.6)$ & $1.05(0.2-5.3)$ & $0.6(0.01-4.8)$ & NA & Blood loss-47 ML $P=0.1$ \\
Nitrates & $0.84(0.25-2.3)$ & NA & NA & NA & NA \\
Aspirin & NA & NA & NA & NA & $1.48(0.85-2.57)$ \\
Thoracic epidural & $7.0(4.0-10.0)$ & NA & $0.5(.01-3.0)$ & NA & $0.43(0.28-0.66)$ \\
\hline
\end{tabular}

$\mathrm{OR}=$ odds ratio CI = confidence interval; $\mathrm{NA}=$ data not available.

\section{Blood transfusion}

\begin{abstract}
"Based on the results of this subgroup analysis we suggest that hemodynamically stable, critically ill patients, with cardiovascular disease may receive transfusions safely when the hemoglobin concentrations fall below $70 \mathrm{~g} \cdot \mathrm{L}^{-1}$ and be maintained at hemoglobin concentrations between 70 and 80 $g \cdot L^{-1}$." $^{\prime \prime}$
\end{abstract}

\section{Hebert et al. Critical Care Med 2001.}

The TRICC ${ }^{45}$ trial is presently the best evidence to relate to perioperative transfusion practice. The trial documents liberal to restrictive transfusion practice in critically ill patients. Thirty-five percent of patients in this trial were postoperative patients. The results of this trial show that restrictive use of blood products, with a transfusion trigger of $70 \mathrm{~g} \cdot \mathrm{L}^{-1}$, is associated with a reduced relative risk of in-hospital death $\mathrm{RR}$ 0.79 (95\% CI $0.63-0.99$ ); MI RR 0.25 (95\% CI 0.1-0.9): congestive heart failure RR 0.49 (95\% CI $0.3-0.8$ ) and a suggestion of reduced adult respirato- ry distress syndrome. The investigators subsequently published a subgroup analysis of the patients with prior coronary disease. ${ }^{46}$ The patients in the restrictive group with coronary disease had a mortality rate of $26 \%$. In the liberal group coronary patients had a $20 \%$ mortality. The analysis was underpowered to show a difference of this magnitude. However, when compared to patients without coronary disease the restrictive group had a mortality of $16 \%$ compared to $24 \%$ ( $P$ $=0.01$ ) Viewed in a different light patients in the restrictive group, and with coronary disease had an almost twofold increase in the in-hospital death rate. However, in the liberal transfusion group, there was no difference in the death rate between patients with and without coronary disease.

We disagree with the investigators' interpretation of this result and suggest that the trigger for transfusions in patients with coronary disease should be in the 90 to $100 \mathrm{~g} \cdot \mathrm{L}^{-1}$ range with a target of $100 \mathrm{~g} \cdot \mathrm{L}^{-1}$. Certainly, more investigation is needed in this area. 


\section{Diabetes control}

Van den Berghe et al. ${ }^{47}$ completed a randomized trial of tight glycemic control in 1,258 high-risk surgical patients. High risk was defined as the need to go to intensive care postoperatively. Two thirds of the patients were cardiac. There was no limit on the amount of insulin infused. Target blood glucose was 4.2 to 6.1 . Tight glycemic control resulted in a $48 \%$ reduction in mortality (OR $0.52 ; 95 \%$ CI $0.3-0.8$ ) There was also an almost $50 \%$ reduction in renal failure, and a significant reduction in blood transfused.

Tight glycemic control in all surgical patients should be the standard of practice.

\section{Comment}

There are three major points, which have to be recognized. First, the quality of many studies is poor. For example, of the studies used to recommend persantine thallium screening, 50\% were retrospective, in most there was no evaluator blinding, and routine screening for outcomes was not uniformly applied. Secondly, the randomized controlled studies tend to be small and underpowered to answer key questions. There are very few studies, which have enrolled more than 1,000 patients. We therefore have relied on tools such as meta-analysis to pool multiple small studies. Metaanalysis of multiple small studies tends to overestimate an effect, an effect that is now evident with the reporting of small positive outcomes in the larger beta-blocker trials. A less expensive alternative to the randomized controlled trial, which is now being recognized as helpful, is the large observational risk adjusted database. There are very few large databases at this time. Finally, where there is moderate to good evidence to guide practice, the implementation of the practice at the bedside is resisted and/or slow. Examples of this are most hospitals do not have a protocol for running tight glycemic control. Most hospitals have no way of running a beta-blocker infusion postoperatively. Hospitals which participated in the TRICC trial, do routinely use these results to guide practice.

If we compare the evidence we use to guide practice to that of cardiology for instance, the difference is obvious. Cardiologists have a myriad of large, randomized trials to guide practice, in many areas. We, as specialists in perioperative medicine, need to be proactive in demanding better quality of evidence. This means taking the lead in the design of large simple randomized trials and creating large national databases. The POISE trial is the first step and the lead investigators need to be congratulated. It will be our responsibility to incorporate those findings into practice.

\section{Recommendations}

The following recommendations are made, mindful of the limitations of the present data. The one sure conclusion of a full survey of the evidence is the need for more and better information.

The evidence supports preoperative risk stratification. The RCRI seems to be the most accurate and easiest index to remember. After identification of patients with a score of greater than 2 , further stratification should take place using a non-invasive stress test. Wider use of dobutamine stress echocardiography should be employed. Identification of patients with two or more new regional wall motion artifacts should initiate coronary artery angiography.

Patients with left main disease and or poor ventricular function should be sent for revascularization. There are no data to suggest the best mode of treatment in this aspect of care.

Medical therapy should employ combination therapy (a need for further investigation is clear). The therapy should start with sympatholysis (beta-blockers, alpha agonists, or thoracic epidural). The use of diltiazem should also be considered where adequate heart rate and blood pressure could not be obtained by other means. Patients at higher risk, without contraindications, should also receive a statin. Finally ASA in low doses should be initiated perioperatively.

The anesthetic should not use nitrous oxide. Care should be taken to control hyperglycemia. Transfusion triggers of $100 \mathrm{~g} \cdot \mathrm{L}^{-1}$ should be rigorously adhered to.

Postoperative analgesia should employ an epidural wherever possible. The use of NSAIDS, as an adjunct to analgesia, should be withheld until better evidence is obtained.

"The good news is that we have moved from risk assessment to risk reduction. However much work remains to evaluate various medical therapies and integrate them with strategies for coronary revascularization in highest risk patients".

Dr. Lee Goldman "Evidence based perioperative risk reduction" Am J Med 2003; 114: 765.

\section{References}

1 Lie SA, Engesaeter LB, Havelin LI, Furnes O, Vollset SE. Early postoperative mortality after 67,548 total hip replacements: causes of death and thromboprophylaxis in 68 hospitals in Norway from 1987 to 1999. Acta Orthop Scand 2002; 73: 392-9.

2 Fleisher LA, Corbett W, Berry C, Poldermans D. Costeffectiveness of differing perioperative beta-blockade strategies in vascular surgery patients. J Cardiothorac Vasc Anesth 2004; 18: 7-13. 
3 Landesberg G, Shatz V, Akopnik I, et al. Association of cardiac troponin, $\mathrm{CK}-\mathrm{MB}$, and postoperative myocardial ischemia with long-term survival after major vascular surgery. J Am Coll Cardiol 2003; 42: 1547-54.

4 Lee TH, Marcantonio ER, Mangione CM, et al. Derivation and prospective validation of a simple index for prediction of cardiac risk of major noncardiac surgery. Circulation 1999; 100: 1043-9.

5 Rohde LE, Polanczyk CA, Goldman L, Cook EF, Lee RT, Lee TH. Usefulness of transthoracic echocardiography as a tool for risk stratification of patients undergoing major noncardiac surgery. Am J Cardiol 2001; 87: 505-9.

6 Ouriel K, Green RM, Deweese JA, Varon ME. Outpatient echocardiography as a predictor of perioperative cardiac morbidity after peripheral vascular surgical procedures. J Vasc Surg 1995; 22: 671-7.

7 Halm EA, Browner WS, Tubau JF, Tateo IM, Mangano $D T$. Echocardiography for assessing cardiac risk in patients having noncardiac surgery. Study of Perioperative Ischemia Research Group. Ann Intern Med 1996; 125: 433-41.

8 Kertai MD, Bountioukos M, Boersma E, et al. Aortic stenosis: an underestimated risk factor for perioperative complications in patients undergoing noncardiac surgery. Am J Med 2004; 116: 8-13.

9 Shaw LJ, Eagle KA, Gersh BJ, Miller DD. Meta-analysis of intravenous dipyridamole-thallium-201 imaging (1985 to 1994) and dobutamine echocardiography (1991 to 1994) for risk stratification before vascular surgery. J Am Coll Cardiol 1996; 27: 787-98.

10 Etchells E, Meade M, Tomlinson G, Cook D.

Semiquantitative dipyridamole myocardial stress perfusion imaging for cardiac risk assessment before noncardiac vascular surgery: a meta-analysis. J Vasc Surg 2002; 36: 534-40.

11 Mantha S, Roizen MF, Barnard J, Thisted RA, Ellis JE, Foss J. Relative effectiveness of four preoperative tests for predicting adverse cardiac outcomes after vascular surgery: a meta-analysis. Anesth Analg 1994; 79: 422-33.

12 Rabbat CG, Treleaven DJ, Russell JD, Ludwin D, Cook $D J$. Prognostic value of myocardial perfusion studies in patients with end-stage renal disease assessed for kidney or kidney-pancreas transplantation: a meta-analysis. J Am Soc Nephrol 2003; 14: 431-9.

13 Kertai MD, Boersma E, Sicari R, et al. Which stress test is superior for perioperative cardiac risk stratification in patients undergoing major vascular surgery? Eur J Vasc Endovasc Surg 2002; 24 :222-9.

14 Beattie WS, Wijeysundera DN, Abdelnaem E, McCluskey $S$, Karkouti KK. An evaluation of preoperative coronary angiography in non-cardaic surgery. Can J Anesth
2004; 51: A74 (abstract).

15 Fleisher LA, Skolnick ED, Holroyd KJ, Lehmann HP. Coronary artery revascularization before abdominal aortic aneurysm surgery: a decision analytic approach. Anesth Analg 1994; 79: 661-9.

16 Mason JJ, Owens DK, Harris RA, Cooke JP, Hlatky $M A$. The role of coronary angiography and coronary revascularization before noncardiac vascular surgery. JAMA 1995; 273: 1919-25.

17 Hassan SA, Hlatky MA, Boothroyd DB, et al. Outcomes of noncardiac surgery after coronary bypass surgery or coronary angioplasty in the Bypass Angioplasty Revascularization Investigation (BARI). Am J Med 2001; 110: 260-6.

18 Landesberg G, Mosseri M, Shatz V, et al. Cardiac troponin after major vascular surgery: the role of perioperative ischemia, preoperative thallium scanning, and coronary revascularization. J Am Coll Cardiol 2004; 44: 569-75.

19 Kaluza GL, Joseph J, Lee JR, Raizner ME, Raizner AE. Catastrophic outcomes of noncardiac surgery soon after coronary stenting. J Am Coll Cardiol 2000; 35: 1288-94.

20 Posner KL, Van Norman GA, Chan V. Adverse cardiac outcomes after noncardiac surgery in patients with prior percutaneous transluminal coronary angioplasty. Anesth Analg 1999; 89: 553-60.

21 Wijeysundera DN, Beattie WS, Djaiani G, Rao V, Borger M, Karkouti KK. Off-Pump coronary artery surgery for the reduction of maortality and morbidity: a meta-analysis. Can J Anesth 2004; 51: A46 (abstract).

22 Wallace A, Layug B, Tateo I, et al. Prophylactic atenolol reduces postoperative myocardial ischemia. McSPI Research Group. Anesthesiology 1998; 88: 7-17.

23 Poldermans D, Boersma E, Bax JJ, Thomson IR, et al. The effect of bisoprolol on perioperative mortality and myocardial infarction in high-risk patients undergoing vascular surgery. Dutch Echocardiographic Cardiac Risk Evaluation Applying Stress Echocardiography Study Group. N Engl J Med 1999; 341: 1789-94.

24 Stevens RD, Burri H, Tramer MR. Pharmacologic myocardial protection in patients undergoing noncardiac surgery: a quantitative systematic review. Anesth Analg 2003; 97: 623-33.

25 Auerbach AD, Goldman L. B-Blockers and reduction of cardiac events in noncardiac surgery: scientific review. JAMA 2002; 287: 1435-44.

26 Yang H, Raymer K, Bultler R, Parlow J. Metoprolol after vascular surgery: MaVS. Can J Anesth 2004; 51 : A7 (abstract).

27 Giles JW, Sear JW, Foex P. Effect of chronic beta-blockade on peri-operative outcome in patients undergoing 
non-cardiac surgery: an analysis of observational and case control studies. Anaesthesia 2004; 59: 574-83.

28 Crystal E, Thorpe KE, Connolly SJ, et al. Metoprolol prophylaxis against postoperative atrial fibrillation increases length of hospital stay in patients not on preoperative beta blockers: the beta blocker length of stay (BLOS) trial. Heart 2004; 90: 941-2.

29 Wijeysundera DN, Naik JS, Beattie WS. Alpha-2 adrenergic agonists to prevent perioperative cardiovascular complications: a meta-analysis. Am J Med 2003; 114 : 742-52.

30 Wallace AW, Galindez D, Salahieh A, et al. Effect of clonidine on cardiovascular morbidity and mortality after noncardiac surgery. Anesthesiology 2004; 101 : 284-93.

31 Park J, Forrest J, Kolesar R, Bhola D, Beattie S, Chu C. Oral clonidine reduces postoperative PCA morphine requirements. Can J Anaesth 1996; 43: 900-6.

32 Wijeysundera DN, Beattie WS. Calcium channel blockers for reducing cardiac morbidity after noncardiac surgery: a meta-analysis. Anesth Analg 2003; 97: 634-41.

33 Ferguson GG, Sackett DL, Thorpe KE, et al. Low-dose and high-dose acetylsalicylic acid for patients undergoing carotid endarterectomy: a randomised controlled trial. ASA and Carotid Endarterectomy (ACE) Trial Collaborators. Lancet 1999; 353: 2179-84.

34 Anonymous. Prevention of pulmonary embolism and deep vein thrombosis with low dose aspirin: Pulmonary Embolism Prevention (PEP) trial. Lancet 2000; 355: 1295-1302.

35 Robless P, Mikhailidis DP, Stansby G. Systematic review of antiplatelet therapy for the prevention of myocardial infarction, stroke or vascular death in patients with peripheral vascular disease. Br J Surg 2001; 88: 787-800.

36 Lindenauer PK, Pekow P, Wang K, Gutierrez B, Benjamin EM. Lipid-lowering therapy and in-hospital mortality following major noncardiac surgery. JAMA 2004; 291: 2092-9.

37 Kertai MD, Boersma E, Westerhout CM, et al. A combination of statins and beta-blockers is independently associated with a reduction in the incidence of perioperative mortality and nonfatal myocardial infarction in patients undergoing abdominal aortic aneurysm surgery. Eur J Vasc Endovasc Surg 2004; 28: 343-52.

38 Durazzo AE, Machado FS, Ikeoka DT, et al. Reduction in cardiovascular events after vascular surgery with atorvastatin: a randomized trial. J Vasc Surg 2004; 39: 967-75.

39 Breslow MJ, Jordan DA, Christopherson R, et al. Epidural morphine decreases postoperative hypertension by attenuating sympathetic nervous system hyper- activity. JAMA 1989; 261: 3577-81.

40 Ballantyne JC, Carr DB, deFerranti S, et al. The comparative effects of postoperative analgesic therapies on pulmonary outcome: cumulative meta-analyses of randomized, controlled trials. Anesth Analg 1998; 86: 598-612.

41 Beattie WS, Badner NH, Choi P. Epidural analgesia reduces postoperative myocardial infarction: a metaanalysis. Anesth Analg 2001; 93: 853-8.

42 Block BM, Liu SS, Rowlingson AJ, Cowan AR, Cowan $J A J r, W u C L$. Efficacy of postoperative epidural analgesia: a meta-analysis. JAMA 2003; 290: 2455-63.

43 Hohner P, Backman C, Diamond G, et al. Anaesthesia for abdominal aortic surgery in patients with coronary artery disease. Part II: Effects of nitrous oxide on systemic and coronary haemodynamics, regional ventricular function and incidence of myocardial ischaemia. Acta Anaesthesiol Scand 1994; 38: 793-804.

44 Badner NH, Beattie WS, Freeman D, Spence JD. Nitrous oxide-induced increased homocysteine concentrations are associated with increased postoperative myocardial ischemia in patients undergoing carotid endarterectomy. Anesth Analg 2000; 91: 1073-9.

45 Hebert PC. Anemia and red cell transfusion in critical care. Transfusion Requirements in Critical Care Investigators and the Canadian Critical Care Trials Group. Minerva Anestesiol 1999; 65: 293-304.

46 Hebert PC. The TRICC trial: a focus on the sub-group analysis. Vox Sang 2002; 83(Suppl 1): 387-96.

47 Van den Berghe G, Wouters P, Weekers F, et al. Intensive insulin therapy in the critically ill patients. N Engl J Med 2001; 345: 1359-67. 